Research Article

\title{
Existence and Uniqueness of Positive Solutions for a Class of Nonlinear Fractional Differential Equations with Singular Boundary Value Conditions
}

\author{
Yan Debao \\ School of Mathematics and Statistics, Heze University, Heze 274000, Shandong Provence, China \\ Correspondence should be addressed to Yan Debao; bbs0415@yeah.net
}

Received 16 November 2020; Accepted 2 March 2021; Published 31 March 2021

Academic Editor: Giovanni Falsone

Copyright ( 2021 Yan Debao. This is an open access article distributed under the Creative Commons Attribution License, which permits unrestricted use, distribution, and reproduction in any medium, provided the original work is properly cited.

This paper focuses on a singular boundary value (SBV) problem of nonlinear fractional differential (NFD) equation defined as follows: $D_{0^{+}}^{\beta} v(\tau)+f(\tau, v(\tau))=0, \tau \in(0,1), v(0)=v^{\prime}(0)=v^{\prime \prime}(0)=v^{\prime \prime}(1)=0$, where $3<\beta \leq 4, D_{0^{+}}^{\beta}$ is the standard Riemann-Liouville fractional (RLF) derivative. The nonlinear function $f(\tau, v(\tau))$ might be singular on the spatial and temporal variables. This paper proves that a positive solution to the SBV problem exists and is unique, taking advantage of Green's function through a fixed-point (FP) theory on cones and mixed monotone operators.

\section{Introduction}

Last few decades, significant progress has been achieved in solving NFD equations led by both the advance of fractional calculus and its applications to physics, mechanics, chemistry, economics, engineering, and other fields [1-8].

The initial value problem and the boundary problem were studied in [9-21], respectively. The SBV problem was recently studied for NFD [22-33], mostly focused on investigating the positive solution. They were mainly based on nonlinear analysis techniques such as Leray-Schuader theory, FP, topological theories, and mixed monotone method [34-39].

In [22], the presence of positive solutions for the SVB problem for NFD equation was investigated, which is defined as

$$
\left\{\begin{array}{l}
{ }^{C} D_{0^{+}}^{\alpha} v(\tau)+f(\tau, v(\tau))=0, \quad \tau \in(0,1) \\
v(0)=v^{\prime}(0)=v^{\prime \prime}(0)=0
\end{array}\right.
$$

where $2<\alpha \leq 3,{ }^{C} D_{0^{+}}^{\alpha}$ is the standard Caputo's fractional derivative, and $f:(0,1] \times[0,+\infty) \longrightarrow[0,+\infty)$ with $\lim _{\tau \longrightarrow 0^{+}} f(\tau, \cdot)=+\infty$. Assuming some hypotheses on $f$, they gained positive solutions through the nonlinear
Leray-Schauder-type alternative in a cone and the Guo-Krasnoselskii FP theory.

$\mathrm{Xu}$ [28] investigated the following SBV problem for NFD:

$$
\left\{\begin{array}{l}
D_{0^{+}}^{\alpha} v(\tau)=f(\tau, v(\tau)), \quad \tau \in(0,1) \\
v(0)=v(1)=v^{\prime}(0)=v^{\prime}(1)=0
\end{array}\right.
$$

where $3<\alpha \leq 4, D_{0^{+}}^{\alpha}$ represents the standard RLF derivative, and $f:[0,1] \times(0,+\infty) \longrightarrow[0,+\infty)$ with $\lim _{v \longrightarrow 0^{+}} f(\cdot, v)$ $=+\infty$. They obtained the presence of multiple solutions under the condition of $f(\tau, v)=g(v)+h(v)$ and the uniqueness of solution for $f(\tau, v)=q(\tau)[g(v)+h(v)]$ using the Guo-Krasnoselskii FP theory, mixed monotone scheme, and Leray-Schauder's nonlinear alternative.

Zhang and Zhong [31] investigated the boundary value problem of singular NFD written as

$$
\left\{\begin{array}{l}
D_{0^{+}}^{\alpha} v(\tau)+f(\tau, v(\tau))=0, \quad \tau \in(0,1), \\
v(0)=0, D_{0^{+}}^{\beta} v(0)=0, D_{0^{+}}^{\beta} v(1)=\sum_{i=1}^{n} \xi_{i} D_{0^{+}}^{\beta} v(\eta),
\end{array}\right.
$$

where the function $f$ permits singularities at $\tau=0, \tau=1$, and $v=0$. The presence of multiple positive solutions for $f(\tau$, 
$v) \leq a(\tau) g(v)+h(v)$ was proved through the Guo-Krasnoselskii FP theory.

In this paper, motivated by those works, the following SBV problem for NFD is investigated:

$$
\begin{aligned}
& D_{0^{+}}^{\beta} v(\tau)+f(\tau, v(\tau))=0, \quad \tau \in(0,1), \\
& v(0)=v^{\prime}(0)=v^{\prime \prime}(0)=v^{\prime \prime}(1)=0,
\end{aligned}
$$

where $3<\beta \leq 4, D_{0^{+}}^{\alpha}$ is the standard RLF derivative, and $f(\tau, v(\tau))$ becomes singular when $\tau=0, \tau=1$, and $v=0$. In analogy with other works, the corresponding Green's NFD and its positive characteristics are inferred. As application, the presence of multiple positive solutions of (4) for $f(\tau$, $v) \leq a_{1}(\tau) f_{1}(v)+a_{2}(\tau) f_{2}(v)$ and the uniqueness of positive solution of $(4)$ for $f(\tau, v)=a_{1}(\tau) f_{1}(v)+a_{2}(\tau) f_{2}(v)$ are investigated, respectively.

Unlike the existing studies, two novel features are induced in this paper. (1) To the best of our knowledge, boundary condition (5) is firstly considered for NFD. (2) Conditions (H2) and (H5) imposed on $f$ are different from those in [26-34].

The remainder of this paper is structured as follows. Preliminaries are given in Section 2, including definitions, lemmas, the deduction of Green's function for problem (4), and new positive properties. Section 3 proves the presence of positive solutions of (4) by the Guo-Krasnoselskii FP theory and demonstrates an example. Section 4 discusses the uniqueness of the positive solution of (4) by a mixed monotone operator and demonstrates another example.

\section{Preliminaries}

The lemmas and definitions from [3] are given for the convenience of the reader as follows:

Definition 1 (see [3]). The RLF integral of the order $\beta>0$ of a function $f(x):(0,+\infty) \longrightarrow R$ is formulated:

$$
I_{0^{+}}^{\beta} f(x)=\frac{1}{\Gamma(\beta)} \int_{0}^{x}(x-s)^{\beta-1} f(s) \mathrm{d} s,
$$

provided the right side is pointwise defined on $(0,+\infty)$.

Definition 2 (see [3]). The RLF derivative of the order $\beta>0$ of a continuous function $f(x):(0,+\infty) \longrightarrow R$ is formulated as

$$
D_{0^{+}}^{\beta} f(x)=\frac{1}{\Gamma(n-\beta)}\left(\frac{\mathrm{d}}{\mathrm{d} x}\right)^{n} \int_{0}^{x} \frac{f(s)}{(x-s)^{\beta-n+1}} \mathrm{~d} s,
$$

where $n=[\beta]+1$ and $[\cdot]$ represents the integer part of number.

Lemma 1 (see [3]). The solution of the NFD equation defined as

$$
D_{0^{+}}^{\beta} \mu(\tau)=0
$$

is $\mu(\tau)=C_{1} \tau^{\beta-1}+C_{2} \tau^{\beta-2}+\cdots+C_{N} \tau^{\beta-N}, C_{i} \in \mathfrak{R}, \quad i=1,2$ $, \ldots, N$, where $N$ is the smallest integer greater than or equal to $\beta$ and $\mu \in C(0,1) \cap L(0,1)$.

Lemma 2 (see [3]). Under the assumption that $\mu \in C(0,1) \cap$ $L(0,1)$ with a NFD of order $\beta$, then

$$
\left.I_{0^{+}}^{\beta} D_{0^{+}}^{\beta} \mu(\tau)=\mu(\tau)\right) C_{1} \tau^{\beta-1}+C_{2} \tau^{\beta-2}+\cdots+C_{M} \tau^{\alpha-M},
$$

where $C_{i} \in \mathfrak{R}, i=1,2, \ldots, M$, where $M$ is the smallest integer greater than or equal to $\beta$.

Lemma 3. Provided that $\kappa(\tau) \in C[0,1]$ and $3<\beta \leq 4$,

$$
\begin{aligned}
& D_{0^{+}}^{\beta} \mu(\tau)+\kappa(\tau)=0, \quad 0<\tau<1, \\
& \mu(0)=\mu^{\prime}(0)=\mu^{\prime \prime}(0)=\mu^{\prime \prime}(1)=0 .
\end{aligned}
$$

The solution of (10) is unique and as follows:

$$
\mu(\tau)=\int_{0}^{1} G(\tau, s) \kappa(s) \mathrm{d} s
$$

where Green's function $G(\tau, s)$ is denoted as

$$
G(\tau, s)=\frac{1}{\Gamma(\beta)} \begin{cases}\tau^{\beta-1}(1-s)^{\beta-3}-(\tau-s)^{\beta-1}, & 0 \leq s \leq \tau \leq 1, \\ \tau^{\beta-1}(1-s)^{\beta-3}, & 0 \leq \tau \leq s \leq 1 .\end{cases}
$$

Proof. (10) is rewritten as follows through Lemma 2:

$$
\mu(\tau)=-\frac{1}{\Gamma(\beta)} \int_{0}^{\tau}(\tau-s)^{\beta-1} \kappa(s) \mathrm{d} s+C_{1} \tau^{\beta-1}+C_{2} \tau^{\beta-2}+C_{3} \tau^{\beta-3}+C_{4} \tau^{\beta-4}
$$

where $C_{i} \in \mathfrak{R}, i=1,2,3,4$. From the boundary conditions $\mu(0)=\mu^{\prime}(0)=\mu^{\prime \prime}(0)=0$, we have $C_{2}=C_{3}=C_{4}=0$. Then, 


$$
\begin{aligned}
\mu(\tau) & =-\frac{1}{\Gamma(\beta)} \int_{0}^{\tau}(\tau-s)^{\beta-1} \kappa(s) \mathrm{d} s+C_{1} \tau^{\beta-1}, \\
\mu^{\prime}(\tau) & =-\frac{\beta-1}{\Gamma(\beta)} \int_{0}^{\tau}(\tau-s)^{\beta-2} \kappa(s) \mathrm{d} s+C_{1}(\beta-1) \tau^{\beta-2}, \\
\mu^{\prime \prime}(\tau) & =-\frac{(\beta-1)(\beta-2)}{\Gamma(\beta)} \int_{0}^{\tau}(\tau-s)^{\beta-3} \kappa(s) \mathrm{d} s+C_{1}(\beta-1)(\beta-2) \tau^{\beta-3} .
\end{aligned}
$$

By the condition $u^{\prime \prime}(1)=0$, we have

$$
C_{1}=\frac{1}{\Gamma(\beta)} \int_{0}^{1}(1-s)^{\beta-3} \kappa(s) \mathrm{d} s .
$$

Accordingly, the unique solution of problem (10) and (11) is given as

$$
\begin{aligned}
\mu(\tau) & =-\frac{1}{\Gamma(\beta)} \int_{0}^{\tau}(\tau-s)^{\beta-1} \kappa(s) \mathrm{d} s+\frac{\tau^{\beta-1}}{\Gamma(\beta)} \int_{0}^{1}(1-s)^{\beta-3} \kappa(s) \mathrm{d} s \\
& =\int_{0}^{1} G(\tau, s) \kappa(s) \mathrm{d} s .
\end{aligned}
$$

Lemma 3 is proved with this.
Lemma 4. The properties of $G(\tau, s)$ defined by (13) are as follows:

(1) $\tau^{\beta-1} s(2-s)(1-s)^{\beta-3} \leq \Gamma(\beta) G(\tau, s) \leq s(2-s)$

(2) $\tau^{\beta-1} s(1-s)^{\beta-2} \leq \Gamma(\beta) G(\tau, s) \leq \tau^{\beta-1}(1-s)^{\beta-3}, 0 \leq \tau$, $s \leq 1$

(3) $G(\tau, s)>0,0<\tau, s<1$

(4) $G(\tau, s) \in C([0,1] \times[0,1])$

Proof. Property (4) is obvious and (3) holds from (1). Thus, here (1) and (2) will be proved.

(1) When $s \leq \tau$,

$$
\begin{aligned}
\Gamma(\beta) G(\tau, s) & =\tau^{\beta-1}(1-s)^{\beta-3}-(\tau-s)^{\beta-1}=\tau^{2} \tau^{\beta-3}(1-s)^{\beta-3}-(\tau-s)^{\beta-1} \\
& =\left[\tau^{2}-(\tau-\tau s)^{2}\right](\tau-\tau s)^{\beta-3}=\tau^{2} s(2-s)(\tau-\tau s)^{\beta-3} \\
& =\tau^{\beta-1} s(2-s)(1-s)^{\beta-3}, \\
\Gamma(\beta) G_{\tau}(\tau, s) & =(\beta-1) \tau^{\beta-2}(1-s)^{\beta-3}-(\beta-1)(\tau-s)^{\beta-2}, \\
\Gamma(\beta) G_{\tau \tau}(\tau, s) & =(\beta-1)(\beta-2) \tau^{\beta-3}(1-s)^{\beta-3}-(\beta-1)(\beta-2)(\tau-s)^{\beta-3} \\
& =(\beta-1)(\beta-2)\left[(\tau-\tau s)^{\beta-3}-(\tau-s)^{\beta-3}\right] \geq 0 .
\end{aligned}
$$

Obviously, $\Gamma(\beta) G_{\tau \tau}(\tau, s)$ is nondecreasing in $\tau$, and thus, for $0 \leq s \leq \tau \leq 1$, we have

$$
\begin{aligned}
\Gamma(\beta) G(\tau, s) & \leq \max _{0 \leq s \leq \tau \leq 1} \Gamma(\beta) G(\tau, s)=\Gamma(\beta) G(1, s)=(1-s)^{\beta-3}-(1-s)^{\beta-1} \\
& =\left[1-(1-s)^{2}\right](1-s)^{\beta-3}=s(1-s)(2-s)^{\beta-3} .
\end{aligned}
$$

When $0 \leq \tau \leq s \leq 1$, note that $0 \leq s(2-s) \leq 1$, we have

$$
\begin{aligned}
& \Gamma(\beta) G(\tau, s)=\tau^{\beta-1}(1-s)^{\beta-3} \geq \tau^{\beta-1} s(2-s)(1-s)^{\beta-3} \\
& \Gamma(\beta) G(\tau, s)=\tau^{\beta-1}(1-s)^{\beta-3} \leq s^{\beta-1}(1-s)^{\beta-3}=s^{2} \cdot s^{\beta-3} \cdot(1-s)^{\beta-3} \leq s^{2}(1-s)^{\beta-3} .
\end{aligned}
$$

Note that $s(2-s)-s^{2}=2 s-2 s^{2}=2 s(1-s) \geq 0$;

then, 


$$
\Gamma(\beta) G(\tau, s) \leq s(2-s)(1-s)^{\beta-3} . \quad \text { (21) } \quad \text { (2) When } s \leq \tau \text {, }
$$

From (18)-(21), we have the first conclusion in Lemma 4 which holds.

$$
\begin{aligned}
\Gamma(\beta) G(\tau, s) & =\tau^{\beta-1}(1-s)^{\beta-3}-(\tau-s)^{\beta-1}=\tau^{2} \tau^{\beta-3}(1-s)^{\beta-3}-(\tau-s)^{\beta-1} \\
& =\tau^{2}(\tau-\tau s)^{\beta-3}-(\tau-s)^{\beta-1} \geq \tau^{2}(\tau-\tau s)^{\beta-3}-(\tau-\tau s)^{\beta-1} \\
& =\left[\tau^{2}-(\tau-\tau s)^{2}\right](\tau-\tau s)^{\beta-3}=\tau^{2} s(2-s)(\tau-\tau s)^{\beta-3} \\
& =\tau^{\beta-1} s(2-s)(1-s)^{\beta-3}, \\
\Gamma(\beta) G(\tau, s) & =\tau^{\beta-1}(1-s)^{\beta-3}-(\tau-s)^{\beta-1} \leq \tau^{\beta-1}(1-s)^{\beta-3} .
\end{aligned}
$$

When $\tau \leq s$,

$$
\Gamma(\beta) G(\tau, s)=\tau^{\alpha-1}(1-s)^{\beta-3} .
$$

Since $0 \leq s(2-s) \leq 1$ for $0 \leq s(2-s) \leq 1$, so

$\Gamma(\beta) G(\tau, s)=\tau^{\beta-1}(1-s)^{\beta-3} \geq \tau^{\beta-1} s(2-s)(1-s)^{\beta-3}$.

Also,

$$
\Gamma(\beta) G(\tau, s)=\tau^{\beta-1}(1-s)^{\beta-3} .
$$

From (22)-(26), we have the second conclusion in Lemma 4 which holds.

Now, we give the following definitions and lemmas (see [34-39]), which are essential in proving the results.

Lemma 5. For a Banach space $\Psi$, let $\Lambda \subset \Psi$ denote a normal cone in $\Psi$ and $\Phi_{1}$ and $\Phi_{2}$ denote open subsets of $\Psi$ with $\theta \epsilon$ $\Phi_{1} \subset \overline{\Phi_{2}}$. Then, let a completely continuous operator $\mathscr{F}: \Lambda$ $\longrightarrow \Lambda$ satisfy either $\|\mathscr{F} \rho\| \leq\|\rho\|, \rho \in \Lambda \cap \partial \Phi_{1},\|\mathscr{F} \rho\| \geq\|\rho\|, \rho \in$ $\Lambda \cap \partial \Phi_{2}$ or $\|\mathscr{F} \rho\| \geq\|\rho\|, \rho \in \Lambda \cap \partial \Phi_{1},\|\mathscr{F} \rho\| \leq\|\rho\|, \rho \in \Lambda \cap \partial \Phi_{2}$.

Then, $\mathscr{F}$ has an FP in $\Lambda \cap \bar{\Phi}_{2} / \Phi_{1}$.

Let $\varsigma \in \Lambda$ with $\|\varsigma\| \leq 1, \varsigma \neq \theta$ and $Q_{\varsigma}=\{x \in \Lambda \mid x \neq \theta$, there exists constants $m, M>0$, such that $m \varsigma \leq x \leq M \varsigma\}$, where $\theta$ is the zero element in $\Lambda$.

Definition 3. $A: Q_{\varsigma} \times Q_{\varsigma} \longrightarrow Q_{\varsigma}$ is a mixed monotone operator when satisfying the monotone condition of $A(x, y)$ in $x, y \in Q_{\varsigma}$ such that $A\left(x_{1}, y\right) \leq A\left(x_{2}, y\right)$ when $x_{1} \leq x_{2}$ and $A\left(x, y_{1}\right) \leq A\left(x, y_{2}\right)$ when $y_{1} \geq y_{2} . x^{*} \in Q_{\varsigma}$ is an FP of $A$ when $A\left(x^{*}, x^{*}\right)=x^{*}$.
Lemma 6. The unique fixed point $x^{*}$ of $A$ exists when a constant $\gamma \in(0,1)$ satisfies

$$
A\left(\tau x, \frac{1}{\tau} y\right) \geq \tau^{\gamma} A(x, y), \quad \forall x, y \in Q_{\varsigma}, 0<\tau<1,
$$

where $A$ is a mixed monotone operator.

\section{Presence of Positive Solutions of SVB}

The presence and multiplicity of positive solutions of (4) and (5) is investigated here. The nonlinear function $f(\tau, x) \in$ $C((0,1) \times(0,+\infty)),(0,+\infty)), f$ may be singular when $\tau=$ $0, \tau=1$, and $x=0$.

For a Banach space $\Psi=C[0,1]$ with the maximum norm $\max _{0 \leq \tau \leq 1}|\mu(\tau)|$, let $K \in \Psi$ denote a nonnegative cone defined as

$$
K=\left\{\mu \in \Psi \mid \mu(\tau) \geq \tau^{\beta-1}\|\mu\|, \tau \in[0,1]\right\} .
$$

The operator $T$ is defined as follows:

$$
(T \mu)(\tau)=\int_{0}^{1} G(\tau, s) f(s, \mu(s)) \mathrm{d} s, \quad 0 \leq \tau \leq 1 .
$$

Clearly, $T: K \backslash\{\theta\} \longrightarrow C[0,1]$. Denote $B_{r}=\{\mu(\tau)$ $\in \Psi \mid\|\mu(\tau)\|<r\}$ and

$$
K_{r}=\Lambda \cap B_{r}=\left\{\mu \in \Psi \mid \tau^{\beta-1}\|\mu\| \leq \mu(\tau)<r\right\} .
$$

The following are assumed for later use:

$\left(H_{1}\right) f \in C((0,1) \times(0,+\infty),[0,+\infty))$.

$\left(H_{2}\right)$ There exist $a_{1}, a_{2} \in C((0,1),[0,+\infty))$ and $f_{1}, f_{2} \in C((0,+\infty),[0,+\infty))$ satisfying

$$
f(\tau, \mu) \leq a_{1}(\tau) f_{1}(\mu)+a_{2}(\tau) f_{2}(\mu), \quad \forall \tau \in(0,1), \mu \in(0,+\infty)
$$

and for any $r>0$,

$$
\int_{0}^{1}\left[a_{1}(\tau) f_{1 r}(\tau)+a_{2}(\tau) f_{2 r}(\tau)\right] \mathrm{d} t<+\infty,
$$


where $f_{\text {ir }}(\tau)=\max \left\{f_{i}(\mu): r \tau^{\beta-1} \leq \mu \leq r\right\}, i=1,2$.

$\left(H_{3}\right)$ There exist $R_{1}>0,0<c_{1}<(1 / 2)$ and $\left[c_{1}, d_{1}\right] c$ $(0,1)$ and a nonnegative function $b_{1}(\tau) \in L^{1}[0,1]$ with $0<2^{\beta-1}<\int_{c_{1}}^{d_{1}} G(1 / 2, s) b_{1}(s) \mathrm{d} s$ satisfying

$$
f(x, \mu) \geq b_{1}(\tau) \mu, \quad \forall(\tau, \mu) \in\left[c_{1}, d_{1}\right] \times\left(R_{1},+\infty\right) .
$$

$\left(H_{4}\right)$ There exist $\left[c_{2}, d_{2}\right] \subset(0,1)$ and a nonnegative function $b_{2}(\tau) \in L^{1}[0,1]$ with $0<\int_{0}^{1} b_{2}(s) \mathrm{d} s<+\infty$ satisfying

$$
\lim _{\mu \longrightarrow 0^{+}} \inf \min _{\tau \in\left[c_{2}, d_{2}\right]} \frac{f(\tau, \mu)}{b_{2}(\tau)}=+\infty
$$

Lemma 7. For any $r>0, T: K \backslash B_{r} \longrightarrow K$ is completely continuous.

Proof. For any $\mu \in K / B_{r}$, we have $\tau^{\beta-1}\|\mu\| \leq \mu(\tau) \leq\|\mu\|$. From $\left(\mathrm{H}_{2}\right)$ and $(1)$ of Lemma 4

$$
\begin{aligned}
(T \mu)(\tau) & =\int_{0}^{1} G(\tau, s) f(s, \mu(s)) \mathrm{d} s \leq \int_{0}^{1} s(2-s)(1-s)^{\beta-3} f(s, \mu(s)) \mathrm{d} s \\
& \leq \int_{0}^{1} s(2-s)(1-s)^{\beta-3}\left[a_{1}(s) f_{1 r}(s)+a_{2}(s) f_{2 r}(s)\right] \mathrm{d} s<+\infty,
\end{aligned}
$$

meaning that $T$ is well defined.

And, by (1) of Lemma 4,

$$
(T \mu)(\tau)=\int_{0}^{1} G(\tau, s) f(s, \mu(s)) \mathrm{d} s \geq \tau^{\beta-1} \int_{0}^{1} s(2-s)(1-s)^{\beta-3} f(s, \mu(s)) \mathrm{d} s \geq \tau^{\beta-1}(T \mu)(\tau)
$$

So, $T$ maps $K, B_{r}$ into $K$.

For a bounded set $D \in K, B_{r}$, a number $R$ exists such that $R>r$ and $r \leq\|\mu\| \leq R$ for any $\mu \in D$. So, we have

$$
(T \mu)(\tau) \leq \int_{0}^{1} s(2-s)(1-s)^{\beta-3}\left[a_{1}(s) f_{1 r}(s)+a_{2}(s) f_{2 r}(s)\right] \mathrm{d} s<+\infty,
$$

which means $T(D)$ is uniformly bounded. $G(\tau, s)$ is uniformly continuous on $[0,1] \times[0,1]$. Accordingly, $\delta>0$ $\left|G\left(\tau^{\prime}, s\right)-G\left(\tau^{\prime \prime}, s\right)\right|<\frac{\varepsilon}{1+\int_{0}^{1}\left[a_{1}(s) f_{1 r}(s)+a_{2}(s) f_{2 r}(s)\right] \mathrm{d} s}$ $\tau^{\prime}, \tau^{\prime \prime} \in[0,1]$

Consequently,

$$
\begin{aligned}
\left|(T \mu)\left(\tau^{\prime}\right)-(T \mu)\left(\mu^{\prime \prime}\right)\right| & \leq \int_{0}^{1}\left|G\left(\tau^{\prime} s\right)-G\left(\tau^{\prime \prime}, s\right)\right| f(s, \mu(s)) \mathrm{d} s \\
& \leq \int_{0}^{1}\left|G\left(\tau^{\prime}, s\right)-G\left(\tau^{\prime \prime}, s\right)\right|\left[a_{1}(s) f_{1 r}(s)+a_{2}(s) f_{2 r}(s)\right] \mathrm{d} s \\
& \leq \int_{0}^{1} \frac{\varepsilon}{1+\int_{0}^{1}\left[a_{1}(s) f_{1 r}(s)+a_{2}(s) f_{2 r}(s)\right] \mathrm{d} s}\left[a_{1}(s) f_{1 r}(s)+a_{2}(s) f_{2 r}(s)\right] \mathrm{d} s \\
& <\varepsilon,
\end{aligned}
$$

implying $T(D)$ is equicontinuous. According to the Arzela-Ascoli theorem, $T: K \backslash B_{r} \longrightarrow K$ is compact.
In the following, the continuity of $T$ will be proved. Given $\left\{\mu_{n}\right\} \subset K \backslash B_{r}$ and $\left\|\mu_{n}-\mu_{0}\right\| \longrightarrow 0(n \longrightarrow \infty), R>r$ satisfies 


$$
r \leq\left\|\mu_{n}\right\|<R, \quad n=1,2, \ldots
$$

For $\varepsilon>0, \delta \in(0,1 / 2)$ satisfies through the absolute continuity of integral,

$$
\begin{gathered}
\int_{0}^{\delta} s(2-s)(1-s)^{\beta-3}\left[a_{1}(s) f_{1 r}(s)+a_{2}(s) f_{2 r}(s)\right] \mathrm{d} s<\frac{\varepsilon}{3}, \\
\int_{1-\delta}^{1} s(2-s)(1-s)^{\beta-3}\left[a_{1}(s) f_{1 r}(s)+a_{2}(s) f_{2 r}(s)\right] \mathrm{d} s<\frac{\varepsilon}{3} .
\end{gathered}
$$

Due to the uniform continuity of $f(\tau, \mu)$ on $[\delta, 1-\delta] \times$ $\left[r \tau^{\beta-1}, r\right]$ and $\left\|\mu_{n}-\mu_{0}\right\| \longrightarrow 0(n \longrightarrow \infty), n>N$ exists. Then,

$$
\begin{array}{r}
\left|f\left(\tau, \mu_{n}(\tau)\right)-f\left(\tau, \mu_{0}(\tau)\right)\right|<\frac{\varepsilon}{3 \int_{0}^{1} s(2-s)(1-s)^{\beta-3} \mathrm{~d} s}, \\
\tau \in[\delta, 1-\delta] .
\end{array}
$$

Then,

$$
\begin{aligned}
\left\|T\left(\mu_{n}\right)-T\left(\mu_{0}\right)\right\| \leq & \max _{0 \leq t \leq 1} \int_{0}^{1} G(\tau, s) \mid f\left(s, \mu_{n}(s)-f\left(s, \mu_{0}(s) \mid \mathrm{d} s\right.\right. \\
\leq & \int_{0}^{1} s(2-s)(1-s)^{\beta-3} \mid f\left(s, \mu_{n}(s)-f\left(s, \mu_{0}(s) \mid \mathrm{d} s\right.\right. \\
\leq & 2 \int_{0}^{\delta} s(2-s)(1-s)^{\beta-3}\left[a_{1}(s) f_{1 r}(s)+a_{2}(s) f_{2 r}(s)\right] \mathrm{d} s \\
& +\int_{\delta}^{1-\delta} s(2-s)(1-s)^{\beta-3} \mid f\left(s, \mu_{n}(s)-f\left(s, \mu_{0}(s) \mid \mathrm{d} s\right.\right. \\
& +2 \int_{1-\delta}^{1} s(2-s)(1-s)^{\beta-3}\left[a_{1}(s) f_{1 r}(s)+a_{2}(s) f_{2 r}(s)\right] \mathrm{d} s \\
\leq & \frac{\varepsilon}{3}+\frac{\varepsilon}{3}+\frac{\varepsilon}{3} \\
= & \varepsilon .
\end{aligned}
$$

So, $T$ is continuous. The proof is finished.

Theorem 1. Under the conditions $\left(H_{1}\right)-\left(H_{4}\right)$ and the existence of a positive constant $r>0$ and $\lambda>\beta>0$ such that

$$
\int_{0}^{1}\left[a_{1}(s) f_{1 r}(s)+a_{2}(s) f_{2 r}(s)\right] \mathrm{d} s<2^{\lambda} r,
$$

at least two positive solutions $\mu_{1}$ and $\mu_{2}$ of (4) exists with $0<\left\|\mu_{1}\right\|<2^{\lambda} r<\left\|\mu_{2}\right\|$.

Proof. According to Lemma 7, the complete continuity of $T$ is valid from $K \backslash B_{r}$ into $K$ for any $r>0$. Then, the existence of two fixed points $\mu_{1}$ and $\mu_{2}$ with $0<\left\|\mu_{1}\right\|<2^{\lambda} r<\left\|\mu_{2}\right\|$ is proved here. For any $r>0$ and condition $\left(H_{3}\right)$, we choose

$$
r_{1}>\max \left\{2^{\lambda-1} R_{1}, 2^{\lambda} r\right\} \text {. }
$$

When $\mu \in K$ and $\|\mu\|=r_{1}$, we have

$$
\mu(\tau) \geq \tau^{\beta-1} r_{1} \geq\left(\frac{1}{2}\right)^{\beta-1} r_{1}>R_{1}, \forall \tau \in\left[\frac{1}{2}, 1\right] .
$$

Thus, we get from $\left(\mathrm{H}_{3}\right)$ and (45) and (46):

$$
\begin{aligned}
(T \mu)\left(\frac{1}{2}\right) & =\int_{0}^{1} G\left(\frac{1}{2}, s\right) f(s, \mu(s)) \mathrm{d} s \geq \int_{c_{1}}^{d_{1}} G\left(\frac{1}{2}, s\right) f(s, \mu(s)) \mathrm{d} s \\
& \geq \int_{c_{1}}^{d_{1}} G\left(\frac{1}{2}, s\right) b_{1}(s) \mu(s) \mathrm{d} s \geq \int_{c_{1}}^{d_{1}} G\left(\frac{1}{2}, s\right) b_{1}(s) \mathrm{d} s \cdot\left(\frac{1}{2}\right)^{\beta-1} r_{1} \\
& >2^{\beta-1}\left(\frac{1}{2}\right)^{\beta-1} r_{1}=r_{1} .
\end{aligned}
$$


Therefore,

$$
\|T \mu\|=\max _{\tau \in[0,1]}|(T \mu)(\tau)| \geq\left|(T \mu)\left(\frac{1}{2}\right)\right| \geq r_{1}=\|\mu\|
$$

$$
\forall \mu \in K,\|\mu\|=r_{1} \text {. }
$$

The condition $\left(H_{4}\right)$ guarantees that, for $M=r\left[\int_{c_{2}}^{d_{2}} G\right.$ $\left.(1 / 2, s) b_{2}(s) \mathrm{d} s\right]^{-1}$, there exists $R_{2} \in(0,1)$ satisfying

$$
f(\tau, \mu)>M b_{2}(\tau), \quad \forall(\tau, \mu) \in\left[c_{2}, d_{2}\right] \times\left(0, R_{2}\right] .
$$

Choose

$$
0<r_{2}<\min \left\{r, R_{2}\right\} .
$$

When $\mu \in K$ and $\|\mu\|=r_{2}$, we have

$$
(T \mu)\left(\frac{1}{2}\right)=\int_{0}^{1} G\left(\frac{1}{2}, s\right) f(s, \mu(s)) \mathrm{d} s \geq r\left[\int_{c_{2}}^{d_{2}} G\left(\frac{1}{2}, s\right) b_{2}(s) \mathrm{d} s\right]^{-1} \int_{c_{2}}^{d_{2}} G\left(\frac{1}{2}, s\right) b_{2}(s) \mathrm{d} s=r>r_{2} .
$$

Therefore,

$$
\|T \mu\|=\max _{t \in[0,1]}|(T \mu)(\tau)| \geq\left|(T \mu)\left(\frac{1}{2}\right)\right| \geq r_{2}=\|\mu\|,
$$

$\forall \mu \in K,\|\mu\|=r_{2}$.
For $\mu \in K$, where $\|\mu\|=2^{\lambda} r,\left(H_{2}\right)$, (1) of Lemma 4, and $0<s(2-s)(1-s)^{\beta-3}<1$, it is similar to (35) and (44), one can get

$$
\begin{aligned}
(T \mu)(\tau) & =\int_{0}^{1} G(\tau, s) f(s, \mu(s)) \mathrm{d} s \leq \int_{0}^{1} s(2-s)(1-s)^{\beta-3} f(s, \mu(s)) \mathrm{d} s \\
& \leq \int_{0}^{1} s(2-s)(1-s)^{\beta-3}\left[a_{1}(s) f_{1 r}(s)+a_{2}(s) f_{2 r}(s)\right] \mathrm{d} s \\
& \leq \int_{0}^{1}\left[a_{1}(s) f_{1 r}(s)+a_{2}(s) f_{2 r}(s)\right] \mathrm{d} s \\
& <2^{\lambda} r .
\end{aligned}
$$

Thus, we have

$$
\|T \mu\| \leq\|\mu\|, \quad \forall \mu \in K,\|\mu\|=2^{\lambda} .
$$

From Lemma 5, (48), (52), and (54), two fixed points $\mu_{1}, \mu_{2}$ of $T$ satisfy

$$
0<r_{2} \leq\left\|\mu_{1}\right\|<2^{\lambda} r<\left\|\mu_{2}\right\| \leq r_{1} .
$$

The proof is completed.

Example 1. Consider the following SBV problem:

$$
\left\{\begin{array}{l}
D_{0^{+}}^{(7 / 2)} \mu(\tau)+\frac{\tau^{2}}{2} \mu^{-1}+\frac{33 \Gamma(7 / 2)}{\sqrt{3}-\sqrt{2}} \tau^{-(1 / 2)}(1-\tau)^{-(1 / 2)} \mu=0, \quad 0<\tau<1, \\
\mu(0)=\mu^{\prime}(0)=\mu^{\prime \prime}(0)=\mu^{\prime \prime}(1)=0 .
\end{array}\right.
$$

$\beta=(7 / 2)$ and $f(\tau, \mu)=\left(\tau^{2} / 2\right) \mu^{-1}+((33 \Gamma(7 / 2)) /$ $(\sqrt{3}-\sqrt{2})) \tau^{-(1 / 2)}(1-\tau)^{-(1 / 2)} \mu$. We set $f_{1}(\mu)=\mu^{-1}, a_{1}(\tau)$ $=\left(\tau^{2} / 2\right), \quad f_{2}(\mu)=\mu, \quad$ and $\quad a_{2}(\tau)=((33 \Gamma(7 / 2)) /(\sqrt{3}-$ $\sqrt{2})) \tau^{-(1 / 2)}(1-\tau)^{-(1 / 2)}$. By simple computation, we have $f_{1 r}=r^{-1} \tau^{-(5 / 2)}, f_{2 r}=r$, and

$$
\int_{0}^{1}\left[a_{1}(s) f_{1 r}(s)+a_{2}(s) f_{2 r}(s)\right] \mathrm{d} s=r^{-1}+\frac{33 \Gamma(7 / 2)}{\sqrt{3}-\sqrt{2}} r \pi<+\infty .
$$

So, the conditions $\left(H_{1}\right)$ and $\left(H_{2}\right)$ hold. 
Next, we set $b_{1}(\tau)=a_{2}(\tau), b_{2} \quad(\tau)=a_{1}(\tau), \quad\left[c_{1}, d_{1}\right]=$ $\left[c_{2}, d_{2}\right]=[(1 / 4),(3 / 4)]$. Then, it is obviously $f(\tau, \mu) \geq b_{1}$ $(\tau) \mu, \forall(\tau, \mu) \in\left[c_{1}, d_{1}\right] \times\left(R_{1},+\infty\right)$ for any $R_{1}>0$. By simple computation, we have $\int_{c_{1}}^{d_{1}} G((1 / 2), s) b_{1}(s) \mathrm{d} s=33$. $2^{-(5 / 2)}>2^{(7 / 2)-1}$. And $\lim _{\mu \longrightarrow 0^{+}} \inf \min _{\tau \in[(1 / 4),(3 / 4)]}(f(\tau, \mu) /$ $\left.b_{2}(\tau)\right)=+\infty$. So, the conditions $\left(H_{3}\right)$ and $\left(H_{4}\right)$ also hold. Taking $r=1, \lambda=10.25$, we have by (57)

$$
\int_{0}^{1}\left[a_{1}(s) f_{1 r}(s)+a_{2}(s) f_{2 r}(s)\right] \mathrm{d} s \approx 1084.4<2^{10.25} \cdot 1 .
$$

Consequently, condition (44) holds. Then, from Theorem 1 in Example 1 at least two positive solutions $\mu_{1}$ and $\mu_{2}$ exist with $0<\left\|\mu_{1}\right\|<2^{\lambda} r<\left\|\mu_{2}\right\|$.

\section{Uniqueness of Singular Problem Solution}

By property (2) of Green's function,

$\varsigma(\tau) p(s) \leq \Gamma(\beta) G(\tau, s) \leq \varsigma(\tau) q(s), \quad \tau, s \in[0,1]$,

where $\varsigma(\tau)=\tau^{\beta-1}, p(s)=s(1-s)^{\beta-2}$, and $q(s)=(1-s)^{\beta-3}$.

From Lemma 3 and (59), a solution of (4) $\mu(\tau)$ is

$$
\begin{aligned}
\mu(\tau) & =\int_{0}^{1} G(\tau, s) f(s, \mu(s)) \mathrm{d} s, \quad \tau \in[0,1], \\
\varsigma(\tau) \int_{0}^{1} \frac{p(s)}{\Gamma(\beta)} f(s, \mu(s)) \mathrm{d} s & \leq \mu(\tau) \leq \varsigma(\tau) \int_{0}^{1} \frac{q(s)}{\Gamma(\beta)} f(s, \mu(s)) \mathrm{d} s .
\end{aligned}
$$

Then, $\mu(\tau) \in Q_{\varsigma}$.

For a Banach space $\Psi, \Lambda$ is a normal cone in $\Psi$ when $\Lambda=\{\rho(\tau) \in C[0,1]: \rho(\tau) \geq 0, \forall \tau \in[0,1]\}$.

Throughout this section, the following is assumed:

$$
\left(H_{5}\right) f(\tau, \mu)=a_{1}(t) f_{1}(\mu)+a_{2}(t) f_{2}(\mu), \quad \tau \in(0,1),
$$

where $f_{1}:[0,+\infty) \longrightarrow[0,+\infty)$ is nondecreasing and continuous, $f_{2}:(0,+\infty) \longrightarrow(0,+\infty)$ is nonincreasing and continuous, and $a_{1}(\tau), a_{2}(\tau) \in C((0,1),(0,+\infty))$.

Theorem 2. A unique positive solution $\mu^{*}$ of (4) exists with $\forall \gamma \in(0,1)$ satisfying

$$
\begin{array}{r}
f_{1}(\tau \mu) \geq \tau^{\gamma} f_{1}(\mu), \\
f_{2}\left(\tau^{-1} \mu\right) \geq t^{\gamma} f_{2}(\mu),
\end{array}
$$

where $\tau \in(0,1)$ and $\mu>0$, and $\forall a_{1}(\tau), a_{2}(\tau)$ satisfy

$$
\int_{0}^{1} q(s) \varsigma^{-\gamma}(s)\left[a_{1}(s) f_{1}(1)+a_{2}(s) f_{2}(1)\right] \mathrm{d} s<+\infty .
$$

Proof. Taking the similar process in [34], for $\tau \in(0,1), \mu>0$,

$$
\begin{aligned}
f_{2}(\tau \mu) & \leq \tau^{-\gamma} f_{2}(\mu), \\
f_{2}(\tau) & \leq \tau^{-\gamma} f_{2}(1), \\
f_{2}\left(\tau^{-1}\right) & \geq \tau^{\gamma} f_{2}(1), \\
f_{1}(\tau) & \geq \tau^{\gamma} f_{1}(1), \\
f_{1}(\mu) & \leq \mu^{\gamma} f_{1}(1), \quad \mu>1 .
\end{aligned}
$$

Define

$$
Q_{\varsigma}=\left\{\mu \in \Lambda \mid \frac{1}{M} \varsigma(\tau) \leq \mu(\tau) \leq M \varsigma(\tau), \tau \in[0,1]\right\}
$$

where $\varsigma(\tau)=\tau^{\beta-1}$, and $M>1$ is defined as

$$
M>\max \left\{\left\{\xi \int_{0}^{1} \frac{1}{\Gamma(\beta)} q(s) \varsigma^{-\gamma}(s)\left[a_{1}(s) f_{1}(1)+a_{2}(s) f_{2}(1)\right] \mathrm{d} s\right\}^{1 /(1-\gamma)},\left\{\xi \int_{0}^{1} \frac{1}{\Gamma(\beta)} p(s) \varsigma^{\gamma}(s)\left[a_{1}(s) f_{1}(1)+a_{2}(s) f_{2}(1)\right] \mathrm{d} s\right\}^{-1 /(1-\gamma)}\right\}
$$

For $\mu, \nu \in Q_{\varsigma}$, we define

$$
T_{\xi}(\mu, \nu)(\tau)=\xi \int_{0}^{1} G(\tau, s)\left[a_{1}(s) f_{1}(\mu(s))+a_{2}(s) f_{2}(\nu(s))\right] \mathrm{d} s, \quad \forall \tau \in[0,1]
$$


We firstly show that $T_{\xi}: Q_{\varsigma} \times Q_{\varsigma} \longrightarrow Q_{\varsigma}$. Let $\mu, \nu \in Q_{\varsigma}$, and from (65) and (66), we have then from (69),

$f_{1}(\mu(\tau)) \leq f_{1}(M \varsigma(\tau)) \leq f_{1}(M) \leq M^{\gamma} f_{1}(1), \quad \forall \tau \in(0,1)$

$$
f_{2}(\nu(\tau)) \leq f_{2}\left(\frac{1}{M} \varsigma(\tau)\right) \leq f_{2}\left(\frac{1}{M}\right) \varsigma^{-\gamma}(\tau) \leq M^{\gamma} f_{1}(1) \varsigma^{-\gamma}(\tau) f_{2}(1), \quad \forall t \in(0,1)
$$

So, we have

$$
\begin{aligned}
T_{\xi}(\mu, \nu)(\tau) & \leq \xi \int_{0}^{1} \frac{1}{\Gamma(\beta)} \varsigma(\tau) q(s)\left[a_{1}(s) f_{1}(\mu(s))+a_{2}(s) f_{2}(\nu(s))\right] \mathrm{d} s \\
& \leq M^{\gamma} \varsigma(\tau) \xi \int_{0}^{1} \frac{1}{\Gamma(\beta)} q(s)\left[a_{1}(s) f_{1}(1)+a_{2}(s) \varsigma^{-\gamma}(s) f_{2}(1)\right] \mathrm{d} s \\
& \leq M^{\gamma} \varsigma(\tau) \xi \int_{0}^{1} \frac{1}{\Gamma(\beta)} q(s) \varsigma^{-\gamma}(s)\left[a_{1}(s) f_{1}(1)+a_{2}(s) f_{2}(1)\right] \mathrm{d} s \\
& \leq M^{\gamma} \varsigma(\tau) M^{1-\gamma} \\
& =M \varsigma(\tau), \quad \forall \tau \in[0,1] .
\end{aligned}
$$

And, from (62), (67), and (68),

$$
\begin{aligned}
& f_{1}(\mu(t)) \geq f_{1}\left(\frac{1}{M} \varsigma(\tau)\right) \geq f_{1}\left(\frac{1}{M}\right) \varsigma^{\gamma}(\tau) \geq M^{-\gamma} f_{1}(1) \varsigma^{\gamma}(\tau) f_{1}(1), \quad \forall \tau \in(0,1), \\
& f_{2}(\nu(t)) \geq f_{2}(M \varsigma(\tau)) \geq f_{2}(M) \geq M^{-\gamma} f_{2}(1), \quad \forall \tau \in(0,1),
\end{aligned}
$$

for all $\mu, \nu \in Q_{\varsigma}$. So,

$$
\begin{aligned}
T_{\xi}(\mu, \nu)(\tau) & \geq \xi \int_{0}^{1} \frac{1}{\Gamma(\beta)} \varsigma(\tau) p(s)\left[a_{1}(s) f_{1}(\mu(s))+a_{2}(s) f_{2}(\nu(s))\right] \mathrm{d} s \\
& \geq M^{-\gamma} \varsigma(\tau) \xi \int_{0}^{1} \frac{1}{\Gamma(\beta)} p(s)\left[a_{1}(s) \varsigma^{\gamma}(s) f_{1}(1)+a_{2}(s) f_{2}(1)\right] \mathrm{d} s \\
& \geq M^{-\gamma} \varsigma(\tau) \xi \int_{0}^{1} \frac{1}{\Gamma(\beta)} p(s) \varsigma^{\gamma}(s)\left[a_{1}(s) f_{1}(1)+a_{2}(s) f_{2}(1)\right] \mathrm{d} s \\
& \geq M^{-\gamma} \varsigma(\tau) M^{-(1-\gamma)} \\
& =M^{-1} \varsigma(\tau), \quad \forall \tau \in[0,1] .
\end{aligned}
$$

It is easy to check that $T_{\xi}(\mu, \nu)$ is nondecreasing in $\mu$ and nonincreasing in $\nu$. 
Next, for any $\sigma \in(0,1)$ and $\mu, \nu \in Q_{\varsigma}$, we have

$$
\begin{aligned}
T_{\xi}\left(\sigma \mu, \sigma^{-1} \nu\right)(\tau) & =\xi \int_{0}^{1} G(\tau, s)\left[a_{1}(s) f_{1}(\sigma \mu(s))+a_{2}(s) f_{2}\left(\sigma^{-1} \nu(s)\right)\right] \mathrm{d} s \\
& \geq \xi \int_{0}^{1} G(\tau, s)\left[a_{1}(s) \sigma^{\gamma} f_{1}(\mu(s))+a_{2}(s) \sigma^{\gamma} f_{2}(\nu(s))\right] \mathrm{d} s \\
& =\sigma^{\gamma} T_{\xi}(\mu, \nu)(\tau), \quad \forall \tau \in[0,1] .
\end{aligned}
$$

Lemma 6 accordingly holds. Also, a unique $\mu^{*} \in Q_{\varsigma}$ satisfying $T_{\xi}\left(\mu^{*}, \mu^{*}\right)=\mu^{*}$ exists. Theorem 2 is proved.
Example 2. The following example for SBV is considered here:

$$
\left\{\begin{array}{l}
D_{0^{+}}^{(7 / 2)} \mu(\tau)+\frac{1}{\sqrt[9]{\tau}} \mu^{1 / 3}+\tau \mu^{-(1 / 4)}=0, \quad 0<\tau<1, \\
\mu(0)=\mu^{\prime}(0)=\mu^{\prime \prime}(0)=\mu^{\prime \prime}(1)=0
\end{array}\right.
$$

We let $f_{1}(\mu(\tau))=\mu^{1 / 3}(\tau), \quad a_{1}(\tau)=(1 / \sqrt[9]{\tau}), f_{2}(\mu$ $(\tau))=\mu^{-(1 / 4)}(\tau), a_{2}(\tau)=\tau$, and $\gamma=1 / 3$. Accordingly,

$$
\begin{aligned}
f_{1}(\tau \mu) & =\tau^{1 / 3} \mu^{1 / 3} \geq \tau^{\gamma} f_{1}(\mu), f_{2}\left(\tau^{-1} \mu\right)=\tau^{1 / 4} \mu^{-(1 / 4)} \geq \tau^{\gamma} f_{2}(\mu), \\
\int_{0}^{1} q(s) \varsigma^{-\gamma}(s)\left[a_{1}(s) f_{1}(1)+a_{2}(s) f_{2}(1)\right] \mathrm{d} s & =\int_{0}^{1}(1-s)^{(7 / 2)-3} s^{-((7 / 2)-1) \cdot 1 / 3}\left[s^{-(1 / 9)}+s\right] \mathrm{d} s \\
& =18+\frac{6}{7}<+\infty .
\end{aligned}
$$

So, Theorem 2 is validated, indicating the presence of a unique positive solution $\mu^{*}$.

\section{Data Availability}

Data sharing is not applicable to this article as no datasets were generated or analysed during the current study.

\section{Conflicts of Interest}

The author declares no conflicts of interest.

\section{Authors' Contributions}

The author has read and approved the final manuscript.

\section{References}

[1] K. B. Oldham and J. Spanier, The Fractional Calculus, Academic Press, New York, NY, USA, 1974.
[2] K. Miller and B. Ross, An Introduction to the Fractional Calculus and Fractional Differential Equations, Wiley, New York, NY, USA, 1993.

[3] S. Samko, A. Kilbas, and O. Marichev, Fractional Integrals and Derivatives: Theory and Applications, Gordon \& Breach, Yverdon, Switzerland, 1993.

[4] I. Podlubny, Fractional Differential Equations, Academic Press, San Diego, CA, USA, 1999.

[5] K. Diethelm, The Analysis of Fractional Differential Equations, Springer, Berlin, Germany, 2010.

[6] Y. Zhou, Basic Theory of Fractional Differential Equations, World Scientific, Singapore, 2014.

[7] Y. Zhou, Fractional Evolution Equations and Inclusions: Analysis and Control, Elsevier, Amsterdam, Netherlands, 2015.

[8] A. Kilbas, H. Srivastava, and J. Trujiuo, Theory and Applications of Fractional Differential Equations, Elsevier, Amsterdam, Netherlands, 2006.

[9] Y. Zhou, H. Chen, and L. Huang, "Existence of positive solutions for nonlinear fractional functional differential equations," Computers \& Mathematics with Applications, vol. 64, no. 10, pp. 3456-4367, 2012.

[10] M. Matar, "On existence of positive solution for initial value problem of nonlinear fractional differential equations of order 
$1<\alpha \leq 2$," Acta Mathematica Universitatis Comenianae, vol. 84, no. 1, pp. 51-57, 2015.

[11] Z. Bai, S. Zhang, S. Sun, and C. Yin, "Monotone iterative method for fractional differential equations," Electronic Journal of Differential Equations, vol. 6, pp. 1-8, 2016.

[12] B. Hamid, A. Abdelouaheb, and L. Yamina, "Positive solutions for nonlinear fractional differential equations," Positivity, vol. 21, pp. 1201-1212, 2017.

[13] R. P. Agarwal, M. Benchohra, and S. Hamani, "Boundary value problems for fractional differential equations," Galen Medical Journal, vol. 16, no. 3, pp. 401-411, 2009.

[14] Z. Bai and H. Lü, "Positive solutions for boundary value problem of nonlinear fractional differential equation," Journal of Mathematical Analysis and Applications, vol. 311, no. 2, pp. 495-505, 2005.

[15] S. Zhang, "Positive solutions for boundary-value problems of nonlinear fractional differential equations," Electronic Journal of Differential Equations, vol. 6, pp. 1-12, 2006.

[16] D. Jiang and C. Yuan, "The positive properties of the Green function for Dirichlet-type boundary value problems of nonlinear fractional differential equations and its application," Nonlinear Analysis: Theory, Methods \& Applications, vol. 72, no. 2, pp. 710-719, 2010.

[17] R. P. Agarwal, Y. Zhou, and Y. He, "Existence of fractional neutral functional differential equations," Computers \& Mathematics with Applications, vol. 59, no. 3, pp. 1095-1100, 2010.

[18] V. Lakshmikantham and J. Devi, "Theory of fractional differential equations in a Banach Space," European Journal of Pure and Applied Mathematics, vol. 1, pp. 38-45, 2008.

[19] Z.-L. Han, Y.-Y. Pan, and D.-W. Yang, "The existence and nonexistence of positive solutions to a discrete fractional boundary value problem with a parameter," Applied Mathematics Letters, vol. 36, pp. 1-6, 2014.

[20] W. Feng, S. Sun, X. Li, and M. Xu, "Positive solutions to fractional boundary value problems with nonlinear boundary conditions," Boundary Value Problems, vol. 2014, p. 225, 2014.

[21] B. Li, S. Sun, Y. Li, and P. Zhao, "Multi-point boundary value problems for a class of Riemann-Liouville fractional differential equations," Advances in Difference Equations, vol. 2014, no. 1, p. 151, 2014.

[22] T. Qiu and Z. Bai, "Existence of positive solutions for singular fractional differential equations," Electronic Journal of Differential Equations, vol. 146, pp. 1-9, 2008.

[23] J. Mohamed and S. Bessem, "On positive solutions of a class of singular nonlinear fractional differential equations," Boundary Value Problems, vol. 73, 2012.

[24] R. P. Agarwal, D. O'Regan, and S. Staněk, "Positive solutions for Dirichlet problems of singular nonlinear fractional differential equations," Journal of Mathematical Analysis and Applications, vol. 371, no. 1, pp. 57-68, 2010.

[25] L. Guo and X. Zhang, "Existence of positive solutions for the singular fractional differential equations," Journal of Applied Mathematics and Computing, vol. 44, no. 1-2, pp. 215-228, 2014.

[26] Y. Wu and L. Liu, "Positive properties of the Green function for two-term fractional differential equations and its application," Journal of Nonlinear Sciences and Applications, vol. 10, pp. 2094-2102, 2017.

[27] F. Wang, L. Liu, and Y. Wu, "Iterative unique positive solutions for a new class of nonlinear singular higher order fractional differential equations with mixed-type boundary value conditions," Journal of Inequalities and Applications, vol. 2019, p. 210, 2019.
[28] X. Xu, D. Jiang, and C. Yuan, "Multiple positive solutions for the boundary value problem of a nonlinear fractional differential equation," Nonlinear Analysis: Theory, Methods \& Applications, vol. 71, no. 10, pp. 4676-4688, 2009.

[29] Y. Wang, “The Green's function of a class of two-term fractional differential equations boundary value problems and its applications," Advances in Difference Equations, vol. 2020, p. 80, 2020.

[30] Y. He, "Existence and multiplicity of positive solutions for a class of singular fractional differential equations with integral boundary value conditions," Advances in Difference Equations, vol. 2016, p. 31, 2016.

[31] X. Zhang and Q. Zhong, "Multiple positive solutions for nonlocal boundary value problems of singular fractional differential equations," Boundary Value Problems, vol. 65, 2016.

[32] J. Henderson, R. Luca, and R. Luca, "Existence of positive solutions for a singular fractional boundary value problem," Nonlinear Analysis: Modelling and Control, vol. 2017, no. 1, pp. 99-114, 2017.

[33] X. Zhang and Q. Zhong, "Triple positive solutions for nonlocal fractional differential equations with singularities both on time and space variables," Applied Mathematics Letters, vol. 80, pp. 12-19, 2018.

[34] Z. Cao, D. Jiang, C. Yuan, and D. O’Regan, "Existence and uniqueness of solutions for singular integral equation," Positivity, vol. 12, no. 4, pp. 725-732, 2008.

[35] D. Guo and V. Lakshmikantham, Nonlinear Problems in Abstract Cones, Academic Press, New York, NY, USA, 1988.

[36] D. Guo and V. Lakshmikantham, "Coupled fixed points of nonlinear operators with applications," Nonlinear Analysis: Theory, Methods \& Applications, vol. 11, no. 5, pp. 623-632, 1987.

[37] D. Guo, "Existence and uniqueness of positive fixed point for mixed monotone operators and applications," Analysis and Applications, vol. 46, no. 1-2, pp. 91-100, 1992.

[38] D. Guo, "Fixed points of mixed monotone operators with applications," Applicable Analysis, vol. 31, no. 3, pp. 215-224, 1988.

[39] Z. Zhao, "Existence and uniqueness of fixed points for some mixed monotone operators," Nonlinear Analysis: Theory, Methods \& Applications, vol. 73, no. 6, pp. 1481-1490, 2010. 